\title{
SOLASODINA EM ESPÉCIES DE SOLANUM DO CERRADO DO DISTRITO FEDERAL
}

José L. Mola, Elis Regina de Araujo e Gouvan C. de Magalhães*

Departamento de Química - Universidade de Brasília - 70 910-900 - Brasília - DF

Recebido em 3/7/96; aceito em 12/2/97

\begin{abstract}
SOLASODINE FROM SOLANUM SPECIES OF THE FEDERAL DISTRICT. An isolation method of the steroidal alkaloid solasodine was applied to the fruits of seven species of the Solanum genus from the Federal District. Two of these species show promising yields. The purity of the isolated alkaloid allows it to be transformed it into an intermediate for steroidal drugs production, 16deshydropregnenolone, and to the lactone vespertiline.
\end{abstract}

Keywords: solasodine; alkaloids; steroids.

\section{INTRODUÇÃO}

Os compostos esteroidais apresentam múltiplas variações estruturais. Suas propriedades farmacológicas foram profundamente estudadas e constituem, em nossos dias, um poderoso recurso que encontra aplicações tanto em medicina humana como veterinária. A produção comercial dessas drogas tem tido, fundamentalmente, como matéria prima, a diosgenina, uma sapogenina esteroidal, presente na forma de glicosídeo em várias plantas, e em alta concentração nas dioscoreas ${ }^{1}$. A disponibilidade, no mercado, desta matéria prima vem apresentando, no entanto, dificuldades crescentes e, por isto, foi necessário encontrar outras fontes capazes de substituir a diosgenina. O conhecimento de que o alcaloide esteroidal solasodina, análogo nitrogenado da diosgenina, pode, igualmente a esta, ser convertido no acetato de 16-deshidropregnenolona ${ }^{2}$ despertou, há algum tempo, o interesse em encontrar plantas com alto conteúdo deste alcaloide ${ }^{3,4,5}$, interesse que ainda se mantém vigente $^{6}$ (Fig. 1).
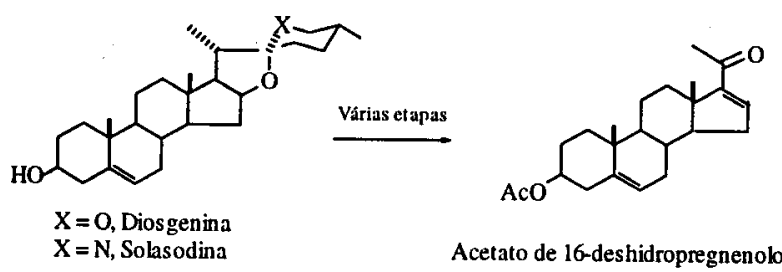

Acetato de 16-deshidropregnenolona

Figura 1

Tanto o colesterol como o $\beta$-sitosterol têm sido ultimamente transformados, por via microbiológica, em compostos que permitem preparar diuréticos e anticoncepcionais. Estas transformações são tão convenientes que a diosgenina deixou de ser a fonte preferencial, de matéria prima, para essas drogas. No entanto, os anticoncepcionais constituem apenas 5\% da produção mundial de esteroides. Progestinas e corticosteroides para tratamento de artrites, inflamações da pele, etc, que representam o grosso da produção mundial de drogas esteroidais, continuam, no entanto, a ser mais facilmente acessíveis a partir da 16-deshidropregnenolona obtida da degradação da diosgenina ou solasodina ${ }^{4}$. Por outro lado, a estrutura da 16 -deshidropregnenolona, por possuir a cadeia acetílica em $\mathrm{C}_{17}$, facilita sua transformação em acetato de deshidroepiandrosterona ${ }^{7,8}$ (Fig.2), material de partida para a produção de andrógenos.

É importante salientar que países como a antiga URSS e
Hungria, durante anos, utilizaram a solasodina, extraída do Solanum laciniatum como única fonte de produção de progesterona e cortisona ${ }^{4,9}$.

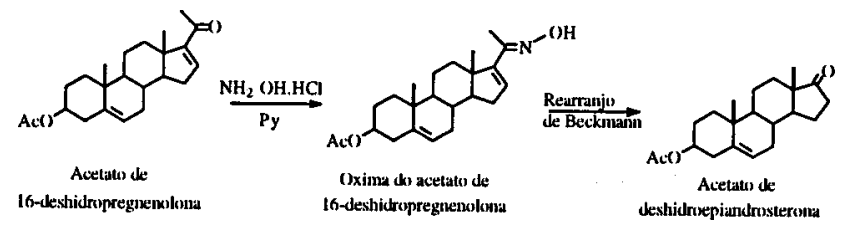

Figura 2

Com frequência, existem marcadas diferenças entre o conteúdo de alcaloide no material vegetal e o que pode realmente ser isolado do mesmo com qualidade conveniente para sua degradação química até a 16-deshidropregnenolona. Submetemos os frutos de sete espécies de solanos, encontrados no DF, a uma técnica de purificação e isolamento, aperfeiçoada no nosso laboratório, que permitiu tirar conclusões quanto à quantidade comprovada de solasodina nos materiais estudados. Este método de extração é especialmente conveniente por permitir o isolamento de solasodina livre do dieno que se forma no processo de hidrólise dos glicosídeos.

\section{RESULTADOS E DISCUSSÃO}

Os resultados alcançados com o estudo químico dos frutos de sete espécies de solanos encontrados no DF, aparecem na tabela a seguir.

Duas espécies de solanos mostraram-se adequadas à exploração comercial, tendo em vista que apresentaram um conteúdo de solasodina maior do que $1 \%{ }^{17}$ : Solanum incarceratum e Solanum viarum. Seria recomendável cultivá-las em áreas maiores e constatar outros parâmetros: possível rendimento por hectare, resistência a pragas e enfermidades, etc. O Solanum lycocarpum apresenta um rendimento de solasodina inferior a $1 \%$, mas pode ter interesse comercial devido ao tamanho dos seus frutos (encontramos frutos com até $1 \mathrm{~kg}$ de peso) e por ser planta nativa do cerrado.

O método de extração da solasodina, dos frutos de plantas do gênero Solanum, por nós desenvolvido, é econômico e mostrouse possível de ser utilizado em quantidades industriais. Ele permite obter solasodina com qualidade adequada para enfrentar transformações posteriores. Os parâmetros exigidos, para estes fins, são um mínimo de pureza de $95 \%$ e conteúdo máximo de $3 \%$ do dieno que é sempre produzido como artefato da hidrólise ${ }^{18}$. 


\begin{tabular}{|c|c|c|c|c|c|c|}
\hline Data & BS (g) & Ppt (g) & $S(g)$ & $\%$ & Média (\%) & Literatura \% \\
\hline 07.09 .94 & 243,10 & 2,5 & 1,70 & 0,69 & & \\
\hline 07.10 .94 & 220,15 & 3,4 & 1,54 & 0,70 & & \\
\hline 07.10 .94 & 440,30 & 7,8 & 2,99 & 0,68 & & \\
\hline 12.11 .94 & 229,65 & 0,9 & 0,58 & 0,25 & & \\
\hline 07.12 .94 & 101,08 & 1,68 & 0,92 & 0,91 & 0,64 & $0,26^{10,11}$ \\
\hline
\end{tabular}

\begin{tabular}{ccccccc}
\hline Data & BS $(\mathbf{g})$ & Ppt $(\mathbf{g})$ & S $($ g) & \% & Média $(\%)$ & Literatura \% \\
\hline 10.03 .95 & 54,14 & 2,48 & 0,89 & 1,65 & & 1,71 \\
07.04 .95 & 133,1 & 6,74 & 2,36 & 1,77 &
\end{tabular}

$\underline{\text { Solanum aculeatissium }}$

\begin{tabular}{ccccccc}
\hline Data & BS $(\mathbf{g})$ & Ppt $(\mathbf{g})$ & S $(\mathbf{g})$ & \% & Média $(\%)$ & Literatura \% \\
\hline $\begin{array}{c}\text { 07.04.95 } \\
\text { Solanum viarum }\end{array}$ & 106,1 & 4,47 & 0,95 & 0,89 & & $2,80^{12}$ \\
\hline Data & BS $(\mathbf{g})$ & Ppt $(\mathbf{g})$ & S $(\mathbf{g})$ & $\%$ & Média $(\%)$ & Literatura \% \\
\hline 22.02 .95 & 37,30 & 1,25 & 0,81 & 2.17 & & \\
07.04 .95 & 68,5 & 2,40 & 1,63 & 2,38 & 2,46 & $5,4^{13} ; 1,2^{14}$ \\
25.04 .95 & 88,16 & 3,56 & 2,49 & 2,82 &
\end{tabular}

$\underline{\text { Solanum grandiflorum }}$

\begin{tabular}{|c|c|c|c|c|c|c|}
\hline Data & BS (g) & Ppt (g) & $S(g)$ & $\%$ & Média (\%) & Literatura \% \\
\hline $\begin{array}{c}04.11 .94 \\
\text { Solanum gem }\end{array}$ & 222,5 & 2,80 & 1,37 & 0,62 & & $0,15^{10}$ \\
\hline
\end{tabular}

\begin{tabular}{ccccccc}
\hline Data & BS $($ g) & Ppt $($ g) & S $($ g) & \% & Média $(\%)$ & Literatura $\%$ \\
\hline 26.06 .95 & 98 & 2,31 & mistura & $\cong 1$ &
\end{tabular}

Solanum asperum

\begin{tabular}{|c|c|c|c|c|c|c|}
\hline Data & BS (g) & Ppt (g) & $\mathbf{S}(\mathbf{g})$ & $\%$ & Média (\%) & Literatura \% \\
\hline 20.11 .95 & 76,57 & 0,54 & mistura & & & $0,42^{16}$ \\
\hline
\end{tabular}

$\mathbf{S}=$ solasodina, $\mathbf{B S}=$ base seca, $\mathbf{P p t}=$ precipitado, Literatura $=$ dados obtidos da literatura.

\section{PARTE EXPERIMENTAL}

Foram coletados frutos de sete espécies de solanos do Distrito Federal, identificados pela professora Carolyn Elionore Barnes Proença e por sua aluna de Mestrado Suelma Ribeiro Silva, do Departamento de Botânica da Universidade de Brasília.

\section{Método de purificação e isolamento de solasodina}

As operações estão referidas a uma quantidade, $200 \mathrm{~g}$, de pó vegetal seco em estufa a $60^{\circ}$.

O pó vegetal, seco, é extraído com etanol $95^{\circ}$ em soxhlet. O extrato alcoólico é concentrado sob vácuo até $66 \mathrm{~mL}$ e a ele é adicionado um volume igual de $\mathrm{HCl} 0,5 \mathrm{M}$. A mistura anterior é lavada com $3 \times 15 \mathrm{~mL}$ de tolueno a temperatura de $65^{\circ} \mathrm{C}$. Uma solução de $\mathrm{NaOH} 6 \mathrm{M}$ é adicionada à mistura quente $\left(65^{\circ}\right)$, até alcançar $\mathrm{pH}=10$. O líquido é esfriado até $15^{\circ} \mathrm{C}$ e é deixado em repouso por 30'. Assim, é obtido um precipitado (glicosídeos crus) que é separado por centrifugação, a 3.000 RPM. O precipitado, glicosídeos, é hidrolisado por refluxo em uma solução de $15 \mathrm{~mL}$ de $\mathrm{EtOH} 95^{\circ}$ e $15 \mathrm{~mL}$ de $\mathrm{HCl} 3,0 \mathrm{M}$, para cada $1,0 \mathrm{~g}$ de sólidos. Uma solução de $\mathrm{NaOH} 6 \mathrm{M}$ é adicionada à mistura, ainda quente, até alcançar pH 10. A mistura é esfriada a $15^{\circ} \mathrm{C}$ e deixada permanecer em repouso por $30^{\prime}$. O precipitado produzido (precipitado primário) é separado por filtração a vácuo, é seco a $70^{\circ} \mathrm{C}$ e pulverizado. O pó é refluxado, durante $20^{\prime}$, em tolueno $(10 \mathrm{~mL}$ para $1,0 \mathrm{~g})$ e filtrado a quente. A solução, de tolueno, é esfriada a $5-8^{\circ} \mathrm{C}$ e o alcaloide cristalizado é separado por filtração a vácuo e lavado com tolueno frio $\left(0\right.$ a $\left.5^{\circ} \mathrm{C}\right)$.

O produto puro, obtido das espécies 1 a 5 , apresentou PF na faixa de $199-201^{\circ} \mathrm{C}$ e foi submetido a análises espectroscópicas de IV, RMN de ${ }^{1} \mathrm{H}$ e EM que se mostraram idênticas às da literatura. As espécies 6 e 7 apresentaram-se como sendo uma mistura de alcaloides de difícil separação, mas foi possível identificar um deles como sendo a solasodina.

As mostras de solasodina obtidas também foram submetidas a duas técnicas de degradação química, descritas na literatura, na produção de 16-deshidropregnenolona ${ }^{19}$ e na produção de 3,16dihidroxi-5-bisnorcolênico $22 \rightarrow 16$ lactona (vespertilina) ${ }^{20,21}$.

\section{AGRADECIMENTOS}

Os autores agradecem à FAPDF e ao RHAE pelo apoio financeiro.

\section{REFERENCIAS}

1. Jaggi, R. K.; Kapoor, V. K.; Indian Drugs 1992, 29, 191.

2. Sato, Y.; Ikekawa, N.; Mosettig, E.; J. Org. Chem. 1959, 24, 893 .

3. Schreiber, K.; In The Alkaloids, Vol. X; Manske, R. H. F., Ed.; Academic Press; New York-London 1968.

4. Mann, J. D.; Advances in Agronomy 1978, 30, 207.

5. Ripperger, H.; Schreiber, K.; In The Alkaloids, Vol. XIX; Rodrigo R. G. A. Ed.; Academic Press; New York-London, 1981.

6. Jaggi, R. K.; Kapoor, V. K.; J. of Scientific \& Industrial Research 1994, 53, 34.

7. Rosenkranz. G.; Mancera, O.; Sondheimer, F.; Djerassi C.; J. Org. Chem. 1994, 21, 520. 
8. Fried, J.; Edwards, J. A.; Organic Reactions in Steroid Chemistry 1972, 21, 520.

9. Verbist, J. F.; Monnet, R.; Plantes médicinales et phytotherapie 1977, tome XI, $n^{\circ} 1,40$.

10. Montidome, M.; Leekning, M. E.; Gottlieb, O. R.; Anais Acad. bras. Ci. 1970, 42 (suplemento), 375.

11. Kerber, V. A.; Miguel, O. G.; Moreira, E. A.; Gomes, E. C.; Weiss, F. A.; Vieira, R. F.; Rev. Bras. Farm. 1993, 74, 77.

12. Kadkade, P.G.; Rolz, C.; Lloydia 1977, 40, 217.

13. Maiti, P. C.; Mookerjea, S.; Mathew, R.; J. Pharm. Sci. 1965, 14, 1828

14. Telek, L.; Plant Med. 1979, 37, 92.

15. Barbosa-Filho, J. M.; Agra, M. F.; Oliveira, R. A. G.; Paulo,
M. Q.; Trolin, G.; Cunha, E. V. L.; Ataide, J. R.; Bhattacharyya, J.; Mem. Inst. Oswaldo Cruz 1991, 86, 189.

16. Bhattacharyya, J.; J. Nat. Prod. 1994, 47, 1059.

17. Kaul, B. L.; Zutshi, U.; In Cultivation and Utilization of Medicinal Plants; Atal, C. K.; Kapur, B. M., ED.; CSIR; New Delhi, 1982.

18. Mola, J; Coll, F.; Fuentes, V.; Granada, M.; Espinisa, J.; Ferrer, A.; Alonso, E.; Rev. Cubana Quim. 1989, 5, 65.

19. Suvorov, N. N.; Morozovkaia, L. M.; Revista de Quimica General 1960, XXX, 2062.

20. Sato, Y.; Latham, H. G.; Mosettig, E.; J. Org. Chem. 1957, 22, 1496.

21. Sato, Y.; Ikekawa, N.; J. Org. Chem. 1961, 26, 5058. 